\title{
¿Una nueva terapia para la política?'1
}

\author{
Profesor Andrew Samuels, $\mathrm{DHL}^{2}$ \\ Londres, U.K.
}

Esta conferencia es una revisión tanto de las posibilidades como de los límites para aquellos analistas y terapeutas que quieran influir en la política, con énfasis en el liderazgo, la desigualdad económica y el cambio climático. También hay una sección sobre los nuevos modelos para las relaciones humanas íntimas (específicamente la promiscuidad y el poliamor) que constituyen una metáfora para la resistencia contemporánea al orden político establecido.

Palabras clave: Psicoterapia, Política, Liderazgo, Cambio Climático, Desigualdad económica.

The talk is a review of the possibilities and limits for analysts and therapists who want to make a difference in politics. There are foci on leadership, economic inequality and climate change. There is also a section on new models for human intimate relations (specifically, promiscuity and polyamory) that constitute a metaphor for contemporary resistance to the established political order.

Key Words: Psychotherapy, Politics, Leadership, Climate Change, Economic Inequality. English Title: A new Therapy for Politics?

\section{Cita bibliográfica / Reference citation:}

Samuels, A. (2018). ¿Una nueva terapia para la política?. Clínica e Investigación Relacional, 12 (1): 3448. [ISSN 1988-2939] [Recuperado de www.ceir.info] DOI: 10.21110/19882939.2018.120103

\footnotetext{
${ }^{1}$ Presentado en las $V$ Jornadas de Psicoanálisis Relacional, Organizadas por el Instituto de Psicoterapia Relacional (IPR), La Granja de San Ildefonso, Segovia, España, 23 de Febrero de 2018. Traducción castellana de María Hernández Gázquez, revisada por Alejandro Ávila y L. Raimundo Guerra.

${ }^{2}$ Andrew Samuels es reconocido internacionalmente como uno de los principales comentaristas político y teórico desde la perspectiva de la psicoterapia y la psicología profunda. Ha trabajado como consultor con líderes políticos, partidos y grupos de activistas en varios países, incluidos Gran Bretaña y los Estados Unidos. También es consultor para el Servicio Nacional de Salud Británico. Basa su trabajo en la experiencia vivida por los ciudadanos, y en lo que se puede aprender del trabajo terapéutico llevado a cabo con una conciencia política. En el ámbito de la clínica, Andrew Samuels aplica un amplio rango de enfoques a la psique, incluidas las ideas post-Jungianas, psicoanalítico relacionales y humanistas. Es Profesor de Psicología Analítica en Essex y profesor visitante en las universidades de Londres, Nueva York, Roehampton y Macau. Es Analista Didacta de la Sociedad de Psicología Analítica de Londres, donde tiene su consulta privada. Andrew fue miembro fundador de la Asociación Internacional de Psicoanálisis y Psicoterapia Relacional, Secretario Honorario de la Asociación Internacional para la Psicología Analítica, y está en la Junta de la revista de psicología humanista Self and Society. Fue presidente del Consejo del Reino Unido para la Psicoterapia (UKCP) y fundador de Psicoterapeutas y Terapeutas para la Responsabilidad Social. Editor consultor fundador de la revista Psychotherapy and Politics International. Sus libros se han traducido a 21 idiomas. Los relevantes para el tema que se aborda aquí son: The Political Psyche (1993), Politics on the Couch (2001), A New Therapy for Politics? (2015), y Analysis and Activism: Social and Political Contributions of Jungian Psychology (co-editado 2016). Otros volúmenes incluyen Jung and the Post-Jungians (1985) [Jung Y Los Post-Jungianos, 2015), The Father (1986) A Critical Dictionary of Jungian Analysis (1986), The Plural Psyche (1989), Psychopathology (1989) y Relational Psychotherapy, Psychoanalysis and Counselling (co-editado 2014). La página web de Andrew es conocida por los 'Andrew's rants' ('los "chorreos" de Andrew') una serie de videos cortos de charlas grabadas: www.andrewsamuels.net
}

CelR Vol. 12 (1) - Febrero 2018 ISSN 1988-2939 - www.ceir.info

(c) Derechos reservados/Copyright de Clínica e investigación Relacional y los autores. Prohibida la reproducción total o parcial sin autorización expresa. Este material es para uso científico y profesional exclusivamente y puede contener información clínica sensible. Los editores no se responsabilizan de los contenidos de los autores. Dirigir las consultas sobre derechos y autorizaciones a ceir@psicoterapiarelacional.es 


\section{INTRODUCCIÓN}

He tenido la extraordinaria suerte de poder trabajar como consultor político con líderes políticos, partidos y grupos de activistas. Estas experiencias incluyen el trabajo en los años 90 sobre cuestiones de liderazgo con Tony Blair y el Partido Laboralista en una época en la cual parecía que el futuro, para él y para el partido, era prometedor. También trabajé en las dos campañas presidenciales de Obama, tanto en 2008 como en 2012. De nuevo, el tema era el liderazgo y lo que un candidato podía hacer con su querida frase 'el padre de la nación'.

También he trabajado con la Coalición para Parar la Guerra, Amigos de la Tierra y con la Alianza de San Jaime ${ }^{1}$ un consorcio de grupos espirituales y de fe dedicados a la justicia social y económica basada en la famosa iglesia de Piccadilly, Londres.

En el campo profesional, he fundado una organización en el Reino Unido llamado Psicoterapeutas y Terapeutas para la Responsabilidad Social y fui co-fundador de la relativamente nueva Unión de Psicoterapia y Terapia. Estos grupos fueron muy activos en las elecciones generales de Junio 2017 en el Reino Unido, debido a que había (y hay) en el país un enorme ataque a los derechos y condiciones de los 'enfermos mentales'. Me avergüenza decir que la corriente predominante en las organizaciones profesionales de terapia es mantenerse en silencio sobre esta cuestión, dejándolo en manos de estas otras agrupaciones más pequeñas y radicales.

Por lo tanto, está claro que soy un entusiasta del interfaz de lo que llamo 'pensamiento terapéutico' y la política. He trabajado en este campo durante más de 40 años, pero justo ahora que podría ser el momento para un 'cambio político' en las terapias, me estoy empezando a preocupar y ser más escéptico. ¿Acaso el cambio político es sólo una moda o una tendencia pasajera? ¿La práctica clínica realmente va a recibir un apoyo político, o seguirá siendo como siempre? Me preocupa que los terapeutas simplemente estén rivalizando unos con otros por el apoyo de una política progresista, olvidando que históricamente nuestros propios políticos profesionales han sido bastante despreciativos con estos temas, y aún hay una enorme personalización y polarización. ¡Espero que mis oyentes sepan esto!

Adicionalmente, no hay buenas estadísticas sobre la aplicación de las psicoterapias, y en particular del psicoanálisis, en relación con las minorías étnicas y sexuales. Hemos estigmatizado y colocado ambas categorías de humanidad en una posición de inferioridad y se podría decir que el cambio hacia el punto de vista opuesto puede constituir el 'Orientalismo', utilizando el término de Edward Said. Esto significa que lo que parece un apoyo y admiración liberal del Otro oprimido, sigue siendo una forma de superioridad colonial que tiene que ver con todo un conjunto de normas del Olimpo. 
En esta corta charla, voy a trabajar sobre tres temas políticos diferenciados: (i) el liderazgo y la responsabilidad; (ii) la desigualdad económica y el cambio climático; y (iii) la política oculta de las relaciones íntimas promiscuas.

De vez en cuando, pediré que realicen una reflexión privada, imaginen algo o evoquen recuerdos sobre cada uno de los tres temas².

\section{EL LIDERAZGO}

Básicamente hay tres tipos diferentes de liderazgo, y pienso que esto es cierto tanto en el caso de la política formal, nacional como dentro de una organización o profesión. Denomino a estos tres tipos de liderazgo como: heroico, de hermandad, y suficientemente bueno. No quiero significar que uno sea mejor que el otro. Sin embargo, dedicaré tiempo al que llamo liderazgo suficientemente bueno ya que esta es mi contribución.

En la teoría y práctica política se ha supuesto que solo hay dos formas principales de abordar el liderazgo. En primer lugar, un liderazgo jerárquico y heroico basado en la autoridad masculina y el enfoque masculino hacia el conocimiento que presume que hay una verdadera historia social objetiva. En este modelo, hay tanto buenos líderes como los hay malos; todos tenemos nuestra propia lista. Weber tenía este tipo de líder en mente cuando en 1924 se refirió al 'líder carismático'. En esta era de Trump, es habitual que este tipo de líder se considere un problema.

El segundo enfoque es mucho más colaborativo, y está relacionado con un modelo de liderazgo, metafóricamente, de hermanos. Está inspirado en el pensamiento feminista. Pero aunque resulte atractivo y a veces sea útil, este tipo de poder es demasiado exigente con los ciudadanos por lo que no resulta operativo para todas las ocasiones. Las personas tienden a "escurrir el bulto" y aunque no necesariamente quieren ser meros observadores, no encuentran otra forma de manejar el peso de ser líderes colaborativos. ¡El trabajo en equipo es duro! Es más fácil dejar que personas destacadas suporten la carga.

Por lo tanto, hay liderazgo heroico y liderazgo colaborativo. Durante muchos años he estado defendiendo un tercer tipo de líder - el líder suficientemente bueno. Es una idea tomada del pensamiento terapéutico sobre la familia. Donald Winnicott le decía a los padres que los bebés tienen que encontrar un punto intermedio entre la idealización y la denigración de los padres. La tendencia natural del bebe es la de idealizar a sus padres, pero cuando las cosas no marchan perfectamente (como suele ocurrir) se convierte en denigración.

¿Le resulta familiar? ¿Una idealización inicial y cuando no se realizan las cosas a la perfección, entonces surge el menosprecio? Debe de resultar familiar. Los medios dependen de ello. Así 
es tal y como respondemos a los líderes, primero pasivamente siguiendo al líder idealizado y luego buscando sus pies de barro. ¿Qué podemos hacer con este patrón?.

Debemos intentar cambiar cómo posicionamos al 'éxito' y el 'fracaso'. Se que la palabra 'fracaso' hiere la sensibilidad de las personas porque es tan evidente. El fracaso significa no llegar, ser imperfecto, falible, solo suficiente, joderla - una falta de poder de sumamente humana. Sin embargo lo que quizás necesitamos hoy en día son políticos del 'no puedo hacer', políticos impotentes - ya lo son de todas formas, tal y como nos demuestran la crisis financiera de finales de 2008 y la incapacidad de tan siquiera imaginar la amenaza al planeta ¿no?.

Quizás no siempre tiene valor solamente el tener las cosas 'bajo control'. Winnicott escribió que 'los padres le fallan al bebé, pero a la manera del propio bebé'. Añadiría que el fracaso de un líder nos abre el camino a que los ciudadanos aporten mayores contribuciones y autonomía. El líder le falla a los ciudadanos, pero a la manera de los propios ciudadanos.

Bob Dylan jugó con el binomio 'éxito-fracaso' al cantar 'no hay éxito comparable al fracaso y el fracaso no es el éxito'. El g de septiembre de 2007 la CNN informó que Bill Clinton hablaba de la 'inevitabilidad' del fracaso política en relación con su plan sanitario. Creo que fue la primera vez que Clinton habló de esa forma tan explícita. Cuando en 1963 un periodista le preguntó a Harold Macmillan, el Primer Ministro Británico, sobre cuál fue el motivo de su caída, simplemente contestó "cosas que pasan, querido chico, cosas que pasan'.

Sin embargo, Rumi escribió en su poema, 'El deseo y la importancia del fracaso' que 'el fracaso es la llave al reino'. Lo suficientemente bueno siempre tiene que ver con el fracaso. La clave es cómo manejar el fracaso, incluso ver el fracaso como un arte; Samuel Beckett escribió que tenemos que 'fracasar mejor'. La desilusión sin duda es difícil, pero también hay que manejarla.

Así que el líder suficientemente bueno puede aceptar la posibilidad del fracaso en una línea del liderazgo post-heroico. Pero hay un problema de cabeza-corazón que viene de un complejo cultural. Con frecuencia sabemos en nuestra mente que el antiguo estilo de líder es peligroso, pero en nuestro corazón y en las entrañas sentimos la necesidad de la protección paternal que ofrece este tipo de liderazgo. En nuestros corazones, estamos enamorados del líder heroico, y nos 'pone' el erotismo tipo 'Führer'. En nuestras cabezas estamos de acuerdo con el Galileo de Brecht: 'descontenta es la tierra que tiene necesidad de héroes'. ¿Podríamos ser más conscientes de la dañina relación amorosa-abusiva que tenemos con los líderes heroicos? 
Definitivamente aquí hay un tema de género. A lo largo de los años, algunas de las respuestas colectivas a Hillary Clinton muestran lo difícil que es que una líder femenina pueda cumplir con el rol de un líder heroico cautivador. Por un lado, es un logro bien recibido ya que, tal y como he estado sugiriendo, con frecuencia entramos en relaciones de abuso con este tipo de líder. Por otro lado, hay un tipo de literalidad y esencialismo en juego en el cual una mujer nunca puede llenar ninguna de las funciones que asociamos al 'padre'. Así que las mujeres líderes tienen que ser más letales que los hombres, como vimos en Bretaña con la Sra. Thatcher. Lo mejor de los líderes suficientemente buenos es que pueden ser líderes suficientemente buenos de cualquier sexo.

Hasta aquí, (suficientemente) bien. Pero ¿qué ocurre con nuestros líderes suficientemente buenos cuando las cosas se ponen violentas? En este punto parece que el liderazgo suficientemente bueno topa con un escollo3. ¿Qué ocurre cuando las cosas se ponen en modo guerra? ¿Dónde nos deja el líder suficientemente bueno con respecto a la acción violenta? Esta cuestión no desaparece cuando se trata de una guerra legitima, guerra ilegitima, estado de miedo y acciones violentamente represivas, o bombardeos suicidas y el culto al mártir.

Entonces ¿acaso no necesitamos las virtudes masculinas? En épocas de 'terror' y guerra sinfín, ¿no tendrán razón los tradicionalistas y conservadores? Acaso ¿no necesitamos la seguridad paternal y la protección militar? ¡Al diablo con el nutrir paternal! Si queréis, esto lo podemos discutir más adelante.

Para finalizar esta sección de la charla me gustaría pediros que reflexionarais sobre los momentos de vuestra vida en la que habéis tenido que tomar las riendas - pero no lo habéis hecho por temor al fracaso.

\section{LA DESIGUALDAD ECONÓMICA Y EL CAMBIO CLIMÁTICO}

Cualquiera, no sólo un terapeuta, que busque mejorar cualquier cosa en el ámbito social se encontrará con masivas fuerzas impersonales y sobre todo económicas que no quieren el cambio, y que están en su contra: El sistema financiero, el patriarcado, y los 'señores' mundiales de la guerra. Pero además hay otro paradójico problema adicional. El inconsciente humano y el alma humana son la fuente de la imaginación, creatividad y esperanza. Sin embargo, en algún grado, también son la fuente de nuestros problemas. En sus aspectos crueles, negativos y sádicos, el inconsciente es, efectivamente, de acuerdo con las enseñanzas de Freud, conservador. Además de resistirse a las mejoras y la transformación, las promociona. Eso mismo que nos da la esperanza de poder encontrar soluciones, es también la fuente de los problemas que suplica soluciones. 
Es posible que en los países desarrollados estemos entrando en un periodo de un enorme desplazamiento tanto de los valores económicos como de la conciencia colectiva - un cambio psicológico profundo y complejo, casi insoportable; quizás condenado a fracasar; de nuestra filosofía de vida - con profundas implicaciones tanto para la sociedad como para el alma.

Sin embargo, dentro este desplazamiento hay claras evidencias dramáticas e inconfundibles de tendencias retrógradas. El incremento de la desigualdad durante los últimos 30 años en países como Estados Unidos y Gran Bretaña se ha constatado repetidamente. También hay constancia del efecto dañino de la desigualdad, ya sea al fomentar el conflicto social e internacional; o en términos de literalmente producir la mala salud (tanto física como mental); o con la producción de culpabilidad en la clase media, uno de los aspectos principales que vincula a los analistas con sus clientes. La excesiva disparidad de riqueza está correlacionada internacionalmente con niveles de enfermedad y mortandad. Desde el punto de vista de una mejor vida, es preferible vivir en un país más pobre pero menos polarizado económicamente, que uno mucho más rico pero muy polarizado.

Deteniéndonos a pensar un momento sobre la desigualdad, está claro que existe una relación entre la clase social y el mundo interno del individuo. Muchos han logrado un status socioeconómico más alto que el de sus padres. Y sin embargo, en sus mundos internos, hallados a través de la terapia y posiblemente de los sueños, la clase social en la que crecieron sigue siendo la clase social en la que están en términos de realidad psíquica y narrativa.

Mi primer cliente banquero soñaba con la mina de carbón en la que había trabajado su padre. La solidaridad (masculina) de los mineros - por ejemplo, cuando había un desastre bajo tierra - le parecía diferente de la atmosfera y el ethos de su gran banco de inversión de Wall Street. Por supuesto jugamos con la pregunta de qué era lo que estábamos 'minando' en su análisis - pero la fuerza principal de nuestro dialogo sobre estos sueños era, en términos de una meticulosa, multi-capa y compasiva cura, una comparativa de su situación completa con la de su padre. No en competición con el padre. El trabajo con este hombre me recordó que en el tema de la relación masculina intergeneracional hay mucho, más allá del Edipo.

El movimiento típico (o al menos lo solía ser, ya que el patrón está cambiando) es desde la clase trabajadora hacia la clase media. En tanto en cuanto exista en la clase trabajadora pasión por la justicia social y económica (por muy buenas razones), se puede ver en algunos la desestabilizante y ego-distónica despiadada escalada a la cumbre. He tenido muchos clientes de este tipo. Este tema de las clases sociales y el mundo interno se aplica con particular fuerza cuando el paciente es miembro de una comunidad de minoría étnica. Lo que no se puede evitar es que posiblemente nos enfrentemos contra una barrera psicodinámica 
ante la movilidad social. La buena noticia es que creo que podemos incidir sobre ello trabajando tanto clínica como culturalmente.

Como un experimento mental teórico, he intentado imaginar una sociedad en la que todos los ingresos se obtienen o devienen de pensiones y la seguridad social, con poca o ninguna propiedad privada del capital. Con altos impuestos sobre la propiedad. Las desigualdades de riqueza se regulan consensualmente. Los mercados se regulan por el compromiso colectivo del bienestar colectivo. Por favor, uniros a mí en imaginar este tipo de economía.

En este experimento de mental surgen las preguntas de si y cómo entraría en juego la desigualdad en el grado en que está presente hoy en las sociedades occidentales. Para algunos, la hipótesis sería que la desigualdad tiene una ineludible base psicológica y que los humanos tienden a encontrar la forma para crear estratificaciones sociales e incluso clases, siendo la clase una forma de adicción 'arquetípica' - o Darwiniana. Señalarían a los colegios británicos en los que todos los alumnos deben utilizar la misma vestimenta según determinan los colegios (llamados 'uniformes') - y aun así la maraña de desigualdades no se pueden excluir ni tan siquiera en el entorno sartorial 4.

En la psicoterapia contemporánea, especialmente la psicoterapia relacional, se podría decir que se busca crear algo parecido a una versión microcósmica de la fantasía con la empecé, en la que la injusticia y desigualdad se reducen lo más posible. Aspiramos a un 'reconocimiento mutuo', de acuerdo con la frase de Jessica Benjamin, o a una inmersión conjunta en un vehículo alquimista, si se prefiere el lenguaje de Jung. Su eslogan fue 'El analista está en tratamiento tanto como el paciente'.

Aquí hay algunas preguntas experienciales en las que espero podáis pensar en privado, mientras estamos aquí:

Permite que empiece preguntando ¿cuáles son tus recuerdos sobre cómo se manejaba el dinero en tu niñez? ¿Son buenos o malos recuerdos?

En tu familia ¿tenía importancia el sexo de una persona en cuanto a los temas de dinero? Por ejemplo, ¿se suponía que los hombres sabían y velaban más por estos temas? ¿Y que las mujeres estaban agradecidas? 0 ¿era al revés?

¿Cómo se movía el dinero en tu familia? ¿Quién controlaba el presupuesto? ¿Se discutía este control en algún momento?

¿Se podía hablar abiertamente de temas de dinero en tu casa? 
¿Has conseguido 'más' que tus padres? En tal caso, ¿ha habido problemas emocionales sobre esto? Por parte de ¿ellos?, ¿tuyo?, o ¿de ambos? Si no has conseguido hacer 'más' ¿esto cómo te hace sentir?

¿Cómo piensas que estás en términos del manejo de los temas económicos en tu actual relación ofamilia? Ponte en una escala de 1-10 donde 1 es muy mal y 10 muy bien.

Cuando tienes fantasías sobre tener mucho dinero, ¿qué haces con él? Si nunca has fantaseado con esto, inténtalo ahora!

Con respecto a esta última pregunta, en los talleres sobre lo que llamo la 'psique económica', les pido a los participantes que tengan fantasías sobre lo más vergonzoso, sádico, controlador y horrible que podrían hacer si tuvieran a su disposición una gran suma de dinero - trillones de dólares. Un profesor en un taller en Pittsburgh dijo, 'Bueno, si tuviera fondos ilimitados, compraría miles de acres de zona de esquí en Aspen y lo vallaría para que nadie lo pudiera utilizar. No me parecía que esto fuera sádico en absoluto. Y entonces espetó: 'Y contrataría a los Marines de Estados Unidos para que ametrallara a cualquiera que se acercara'. Estalló en lágrimas y nos habló de su padre magnate, la relación que tenía con él y más detalles personales.

La vergonzosa fantasía económica nos desvela cómo incluso personas con las opiniones más progresistas están profundamente investidas en un sistema de injusticia económica. Si queremos cambiar este sistema, necesitamos saber reconocer contra qué nos enfrentamos. Tiene que ver con el ser propietarios de nuestro propio pedacito de sistema, un trozo de sombra de la que nos podemos despegar inocentemente. La lección es que el sadismo económico no es algo de lo que se puede escapar simplemente porque se quiera salir del pozo.

Me gustaría ser optimista sobre las perspectivas de justicia económica, pero, sin un cambio de conciencia y el respaldo de muchos grupos -incluidos terapeutas y analistas- hacia un nuevo enfoque de la economía, será difícil lograr este cambio. Los psicoterapeutas pueden contribuir a la idea de que la injusticia económica y la desigualdad económica son malas para la salud mental; malas para el alma y malas para el espíritu.

El futuro humano será mejor y más ligero en cuanto podamos admitir nuestros crímenes económicos personales hacia los demás, otras gentes, sistemas de creencia, géneros y especies. Cuanto más, incluso las clases medias, sigan negando su sadismo económico, mayores serán 'los horrores y venganzas de su tiempo que esperan silenciosamente en los márgenes de los sangrientos dramas de nuestro futuro' (en palabras de Ben Okri). No se trata sólo de la arrogancia de los banqueros. Es la nuestra también. No siendo Ulises, la mayoría 
de nosotros hemos sido seducidos por la facilidad de la deuda personal de Circe. ¿Cuántas tarjetas de crédito tienes en tu cartero o bolso? Como dijo el rabino judío Baal Shem Tov, 'Los pecadores son espejos. Cuando vemos fallos en ellos, debemos reconocer que únicamente son reflejo del mal que hay dentro de nosotros'.

Pienso que la valoración de la igualdad, lo que llamo espiritualidad democrática, no está muerta en la política de países como América, Gran Bretaña o España. Pero el tiempo se está agotando. Ahora, en la crisis financiera sinfín, es hora de hacer un llamamiento al sacrificio económico por parte de los países desarrollados e incluso en nombre de las clases medias de los países donde hay millones por debajo de la línea de la pobreza. Es difícil que los ricos sea vean afectados (aunque fácil de justificar), como ya sabemos. Pero estoy hablando de sacrificios económicos que se podrían hacer por parte de millones de personas normales de clase media. Y si esta idea del sacrificio económico encaja con lo que otros están diciendo sobre la sostenibilidad y el calentamiento global, tanto mejor.

Concluiré esta sección de mi charla con algunas ideas sobre el sacrificio, pensando en el cambio climático y la economía de la sostenible. Sabemos que la gente hace sacrificios por sus hijos o por causas en las que creen, o con la esperanza de un mayor beneficio futuro (lo que los economistas denominan 'coste de oportunidad'). Pero me refiero al sacrificio en y por sí mismo, y los significados espontáneos que puede generar.

El sacrificio es un tema psicológico e histórico muy extendido. El sacrificio yace en el corazón de las religiones Abrahámicas (el sacrificio abortado de Isaac) pero es una apropiación mucho más antigua por parte de los dioses. Tanto el asceticismo como el martirio tienen una larga historia cultural, incluyendo la de los bombarderos suicidas.

La psicología Jungiana hace referencia al sacrificio del ego por el bien de favorecer que florezca a una personalidad más amplia a través de la individualización. En el arte y en la religión, contemplamos el sacrificio de la autonomía y el control por algo vivido como el 'otro', ya sea dentro o fuera del Self. El siguiente y último ejercicio experiencial se ocupará de este tema.

Os pido recordar cuando el renunciar a algo haya producido una inesperada y maravillosa experiencia gratificante. Piensa específicamente en un sacrificio económico, ¿qué sacrificio imaginas que podrías hacer? ¿Qué sacrificios imaginas que podría hacer tu propio país?

\section{LA POLITICA OCULTA DE LA PROMISCUIDAD}

Allá por los años 6o, en el verano del amor, no se hablaba de 'promiscuidad'. La referencia era a 'relaciones no posesivas' o 'familias alternativas' o 'amor libre'. En esta charla, intentaré 
explorar algunas de las implicaciones políticas del divorcio entre el sexo y la relación. Es un tema explosivo y lleno de paradojas.

Es difícil encontrar discusiones contemporáneas sobre la promiscuidad en un contexto occidental que no contengan una perspectiva negativa. La palabra que aparece una y otra vez en el contexto sexual es 'ocasional o esporádico'. Ahora estamos muy familiarizados con el termino sexo ocasional o esporádico.

Antes de seguir, a nivel experiencial, os voy a pedir que penséis en la última vez que experimentaste deseo (definido como se quiera). ¿qué ocurrió? Si actuaste sobre ello, ¿qué sentimientos tenías en ese momento y cuáles ahora? Y si no actuaste sobre tu última experiencia de deseo, ¿qué sentimientos tuviste entonces y cuáles ahora?

\section{La promiscuidad y la política}

Desde finales del siglo XIX la promiscuidad ha sido el fenómeno de trasfondo que sobre el que se han sustentado numerosas discusiones que vinculan a la política con la sexualidad. Los relatos convencionales elogian a las relaciones íntimas cuando éstas irradian constancia, longevidad y fidelidad. Sin embargo los relatos más radicales sugieren que la propiedad y el control sobre el otro son también críticamente importantes. El Origen de la Familia, la Propiedad Privada y el Estado, de Friedrich Engels (1884) es de los más conocidos en el que expone que la oposición de primera clase que aparece en la historia coincide con 'el desarrollo del antagonismo entre hombre y mujer en el matrimonio monógamo' especialmente en 'las clases poseedoras'.

Se puede considerar a la monogamia de hoy como algo que resuena y co-simboliza a los mercados económicos y la posesión implícita y explícita de los recursos planetarios por parte de poderosos países occidentales y de las corporaciones. Se puede argumentar que la monogamia está por tanto implícita en una amplia gama de injusticias - ambientales, económicas y éticas. Ahora bien, este punto se puede hacer con mayor o menor pasión ya que la monogamia desde luego tiene sus méritos y no se puede reducir únicamente al nivel de la tiranía política.

El corolario - que la no-monogamia está correlacionada con la sostenibilidad, igualdad y justicia social - sigue necesariamente sin estar demostrado, aunque sea altamente sugerente. La posesión es una perspectiva tendenciosa tanto en las relaciones como en la geopolítica; pero se puede considerar que las estrategias públicas para la sostenibilidad, tales como el principio de un 'zona común global' co-simbolizan la no-monogamia en la esfera privada. 
Si por ejemplo, consideramos la historia midrashica de Lilith podemos comprender las posibles relaciones entre la política y el comportamiento sexual de forma más fluida. Lilith fue la primera consorte de Adán y fue creada de la tierra en el mismo momento que Adán. No quiso renunciar a su igualdad y discutió con Adán sobre la posición que debían tener durante el coito - Lilith insistiendo que ella debía estar encima. '¿Por qué debo yacer bajo ti', discutía, 'cuando yo soy tu igual ya que ambos fuimos creados del polvo?' Adán estaba decidido y comenzó a violar a Lilith quien invocó el mágico nombre de Dios, subió a los cielos y salió volando. Entonces fue creada Eva. Finalmente la carrera de Lilith - nada sorprendentemente - fue la de diablesa maligna que visita por las noches a los hombres secretamente, siendo por tanto responsable de las poluciones nocturnas. También es asesina de recién nacidos. Pero al final, después de la destrucción del Templo, Lilith entra en relación con Dios como una especie de amante.

Lo que quiero recalcar es que este tipo de material se puede tomar tanto como una expresión de la influencia de lo sexual sobre la política como al revés. La experiencia que las personas tienen de lo sexual también es motor de su politicidad, estilo y valores políticos. La experiencia sexual y sus imágenes asociadas expresan el enfoque psicológico individual del funcionamiento político.

\section{Promiscuidad y espiritualidad}

Habiendo revisado algunos de los problemas con la idea de la promiscuidad y habiendo colocado mis ideas en contextos políticos e históricos, quiero profundizar y darle mayor complejidad a la discusión, al posicionar a la promiscuidad como un fenómeno espiritual.

Es difícil definir lo 'espiritual', pero tiene que ver con algo 'mas-que-personal' que yace sobre, debajo, o bajo o detrás de lo cotidiano. Hay con frecuencia una sensación de estar confrontado con algo impresionante y 'mayor' que uno mismo - más-que-personal. Pero la espiritualidad que informa a la promiscuidad es ubicua, oculta en abierto, algo esperando ser descubierto, no como resultado de una 'venta' de alguien con ambiciones por su religión, secta o culto.

Lo que estoy diciendo es que el comportamiento promiscuo sexual se puede comprender en términos de una experiencia mística. Hay algo numinoso de la experiencia promiscua, como muchos sabéis. La atracción física sobrecogedora produce sentimientos de impresión, asombro y temblor. Hay como un Dios que despierta, un Dios primitivo, ctónico5, temprano y elemental. Hay una experiencia de lo divino sin restricciones. 


\section{Las Promiscuidades}

La promiscuidad no es un monolito - con frecuencia hay desconcertantes diferencias relacionados con el género, la diversidad sexual, clase social y etnia. Ahora bien, es interesante subrayar la frecuencia con que las discusiones sobre la promiscuidad, incluso en círculos de profesionales liberales de psicoterapeutas, caen en discusiones sobre el comportamiento promiscuo de hombres homosexuales. Por tanto, es casi imposible manejar una conversación razonada sobre la promiscuidad.

\section{La Promiscuidad y la imaginación}

Hasta ahora, hemos estado discutiendo los aspectos históricos, políticos y espirituales de la promiscuidad. Sin embargo, como terapeuta, sé que la promiscuidad no es solo un tema literal. También está involucrado en un amplio rango de discursos metafóricos e imaginativos. Además del simbolismo político, tenemos que pensar en la promiscuidad como algo que simboliza la creatividad que rompe fronteras, tanto en sentido artístico como en general (se podría ser promiscuo políticamente, por ejemplo).

Desde un punto de vista psicológico, la promiscuidad llama a las dimensiones simbólicas o metafóricas de los temas de libertad, diferenciación del trasfondo parental y familiar, y una nueva relación con la escena primal (lo que quiere decir la imagen que tenemos en nuestra mente de la vida íntima o ausencia de la misma de nuestros padre(s)). El psicoanálisis Kleniano habla del 'estado de mente de la pareja', los padres en la mente ocupados en fértiles y creativas relaciones sexuales. La intención es proponer un símbolo universal de creatividad, fecundidad y salud mental en general. Sin embargo el resultado es con frecuencia una angustiosa aplicación literal de la idea. Por tanto debemos preguntar: acaso el estado de mente de la pareja, la pareja en la mente, ¿es siempre una pareja estable 'casada' o comprometida? También podríamos preguntar: ¿será siempre una pareja heterosexual? ¿Siempre una pareja de la misma etnia?.

\section{La promiscuidad y la psicoterapia}

Con estas ideas en mente, quiero centrarme ahora en nuestra profesión de psicoterapeutas, tanto en y sobre sí mismo y como representación de una cultura más amplia. Mi acusación es que, en lo que se refiere a la promiscuidad, la psicoterapia como institución y muchos (aunque no todos) los psicoterapeutas como individuos, son hipócritas. En los términos de la etimología de la palabra 'hipocresía'; están actuando o fingiendo algo. Además de apuntarme un tanto, estoy interesado en explorar este fenómeno. 
Es significativa la ausencia de teorización sobre el sexo fuera de la relación, por parte de analistas y terapeutas y que, cuando se toma una postura teórica, invariablemente es en términos de psicopatología, de un supuesto miedo a la intimidad, problemas con las relaciones de apego ('apego ambivalente'), la perversión, etc. Hay una ausencia contemporánea de consideración a lo que mi difunta amiga Muriel Dimen se refería a 'sexocomo-fuerza'.

Creo que es interesante preguntar si puede haber algo en el pensamiento fundamental o del encuadre de la psicoterapia que nos lleve al conservadurismo adverso a la carnalidad. Ciertamente, la proliferación de escuelas en la psicoterapia es una preciosa metáfora para todo este tema: por un lado, históricamente la mayoría de los terapeutas han estado casados de forma monógama con una escuela. Sin embargo, se podría argumentar que el campo en sí es cada vez más amenazantemente promiscuo.

Hemos aprendido, principalmente de Foucault, que, para cada discurso mayoritario es probable que haya un discurso minoritario subyugado. En la psicoterapia - así como en la sociedad - el discurso mayoritario es relacional. Por tanto, el discurso minoritario subyugado será lo opuesto a lo relacional; en el lenguaje de esta charla, promiscuo. Me he preguntado si el silencio de los psicoterapeutas sobre el tema de la promiscuidad refleja algún tipo de horror sexual - por lo tanto, traducen todo en un discurso relacional en el que las 'personas' se escinden del 'sexo'.

Al unir estas ideas - de la hipocresía y el discurso subyugado no-relacional - se expone la secreta moral conservadora de un número de clínicos psicoterapeutas, que se compara con su frecuentemente distinto comportamiento como personas. Podemos empezar a comprender esto más profundamente al verlo como envidia por parte del terapeuta de la experimentación sexual y comportamiento fuera-del-orden que relatan sus clientes.

El tema emerge cuando un psicoterapeuta se encuentra con la infidelidad ('engaño') por parte de sus clientes. Mientras no se niega que algunos terapeutas, especialmente los terapeutas de pareja, entienden la infidelidad como un fenómeno sistémico, el enfoque general psicoterapéutico es que es un síntoma de otra cosa, un problema en el que engaña, normalmente de tipo narcisista. El engañado normalmente siente gran dolor y el que engaña frecuentemente siente gran culpabilidad. Son afectos muy fuertes como para que el terapeuta los pueda sentir con el cliente. Por tanto, quizás no es sorprendente que lo que veamos en la mayoría de estas situaciones sea una valorización contra-resistente de la longevidad relacional y una comprensión totalmente literal del 'objeto constancia' a expensas de una calidad relacional. Si estás en una relación de larga duración, estás en todo caso y a todos los efectos, bien. 


\title{
FINALES
}

Lo que he intentado hacer en esta corta charla es mostrar cómo las aplicaciones del pensamiento terapéutico pueden iluminar algunas de las problemáticas políticas actuales. Los tres que seleccioné fueron liderazgo, desigualdad económica especialmente en relación con el cambio climático y la sostenibilidad, y la política oculta de la promiscuidad.

Reconozco que soy un entusiasta sobre esta forma de proceder a la vez que un escéptico. No todos somos progresistas, de izquierdas o espirituales. Muchos colegas y pacientes no lo son, y no debemos olvidarlo nunca.

Terminaré con un poema de Jerzy Ficowsky que pediré a alguien leer en español. Es un poema del Holocausto, pero para mí, captura esta tensión entre entusiasmo y escepticismo al preguntar - pregunta que incluso está en el título de mi libro, con signo de interrogación ¿puede realmente existir alguna vez una 'nueva terapia para la política'?

\author{
No conseguí salvar \\ ni una sola vida \\ No he sabido detener \\ ni una sola bala \\ Así deambulo por cementerios \\ que no existen \\ Busco palabras \\ que no existen \\ Salgo corriendo \\ para ayudar cuando nadie ha llamado \\ para un rescate tras el evento \\ Quiero llegar a tiempo \\ aunque llegue demasiado tarde
}

\section{REFERENCIAS}

Samuels, Andrew (1989). The Plural Psyche: Personality, Morality and the Father. London

\& New York: Routledge.

Samuels, A. (1993). The Political Psyche. London and New York: Routledge. 
Samuels, Andrew (1998).'Responsibility, in Development through Diversity: The Therapist's Use of Self. London: United Kingdom Council for Psychotherapy.

Samuels, A. (2001). Politics on the Couch: Citizenship and the Internal Life. London ad New York: Karnac, 2001.

Samuels, Andrew (2002). The hidden politics of healing; foreign dimensions of domestic practice. American Imago, 59:4, pp.459-481.

Samuels, A. (2004). A new anatomy of spirituality: clinical and political demands the psychotherapist cannot ignore. Psychotherapy and Politics International 2004; 2(3): 201-211

Samuels, A. (2006). Socially responsible roles of professional ethics: inclusivity, psychotherapy and 'the protection of the public. International Review of Sociology 2006; 16(2): 175-190.

Samuels, A. (2011). Carnal critiques: Promiscuity, Politics, Imagination, Spirituality and Hypocrisy. Paper presented at IARPP Conference, Madrid, June 2011. ${ }^{6}$

Original recibido con fecha: 17/12/2017 Revisado: 21/1/2018 Aceptado: 28/02/2018

NOTAS:

${ }^{1}$ Stop the War Coalition, Friends of the Earth, St James' Alliance

2En este punto el autor comenta que: "El taller de mañana será más experiencial y, sobre todo, interactivo".

3 "Hit a rock"

${ }^{4}$ N.d.T: Según la RAE: Perteneciente o relativo al sastre y a sus actividades. Figuradamente, el esplendor fulgurante de la moda.

${ }^{5}$ N.d.T: relativo a los dioses griegos del inframundo

${ }^{6}$ Las personas interesadas en este trabajo en su versión bilingüe inglés-castellano pueden ser solicitarlo a ceir@psicoterapiarelacional.com como un complemento al presente. 